\title{
Study of $q$-Garnier System by Padé Method
}

\author{
By \\ Hidehito NagaO and Yasuhiko YamadA \\ (Akashi College and Kobe University, Japan)
}

\begin{abstract}
We give a simple form of the evolution equation and a scalar Lax pair for the $q$-Garnier system. Some reductions to the $q$-Painlevé equations and the autonomous case as a generalized QRT system are discussed. Using two kinds of Padé problems on differential grid and $q$-grid, we derive some special solutions of the $q$-Garnier system in terms of the $q$-Appell Lauricella function and the generalized $q$-hypergeometric function.

Key Words and Phrases. Padé method, q-Garnier system, QRT system, q-Appell Lauricella function, Generalized $q$-hypergeometric function.

2010 Mathematics Subject Classification Numbers. 14H70, 33D15, 33D70, 34M55, 37K20, 39A13, 39A20, 41A05, 41A20, 41A21.
\end{abstract}

\section{Introduction}

The Garnier system [3, 6] is known as an important extension of the Painlevé equations to multi-variables. Its $q$-difference analog, the $q$-Garnier system, was formulated by $\mathrm{H}$. Sakai in [24].

There exists a simple method to study the Painlevé/Garnier equations using Padé approximation [32]. In this method, one can obtain the evolution equation, the Lax pair and some special solutions simultaneously, starting from a suitable Padé approximation (or interpolation) problem. This method has been applied $[5,15,16,17,18,19,21,35]$ to various cases of discrete Painlevé equations $[10,23]$.

Our aim is to study the $q$-Garnier system applying the Padé method. We study both the usual (i.e. differential) Padé approximation and the Padé interpolation on $q$-grid, and obtain two kinds of special solutions written in terms of the $q$-Appell Lauricella function and the generalized $q$-hypergeometric function.

In Section 2.1, we introduce a scalar Lax pair and derive the $q$-Garnier equation as the necessary condition for the compatibility. In Section 2.2, the correspondence to the Sakai's matrix Lax form is considered. In Section 2.3, the sufficiency for the compatibility is proved. In Section 2.4, we rewrite the $q$-Garnier system into more explicit (but nonbirational) form. In Section 2.5, we discuss the reductions to the $q$-Painlevé equations of types $D_{5}^{(1)}, E_{6}^{(1)}$ and $E_{7}^{(1)}$. The $E_{7}^{(1)}$ case is new and $D_{5}^{(1)}, E_{6}^{(1)}$ cases are known $[24,26]$. 
In Section 3, we formulate a hyper-elliptic generalization of the QRT system [22, 30]. Then the generalized QRT system is identified as the autonomous limit of the $q$-Garnier system.

In Section 4, we study certain Padé problem on differential grid. In Section 4.1, we show that the solutions of the Padé problem give special solutions of the Lax equation and $q$-Garnier system. In Section 4.2, we derive the explicit expressions of the special solutions in terms of the $q$-Appell Lauricella function [25].

Similarly, in Section 5 we study certain Padé problem on $q$-grid and obtain special solutions in terms of the generalized $q$-hypergeometric function. We note that the higher order $q$-Painlevé system given by Suzuki [29] also has special solutions given in terms of the generalized $q$-hypergeometric function.

\section{A simple form of the $q$-Garnier system}

In this section, we give a reformulation of the evolution equation and the Lax equations of the $q$-Garnier system. In particular, the original Sakai's Lax form by $2 \times 2$ matrix is reformulated in a scalar form.

\subsection{Lax pair and the $q$-Garnier equation}

Fix a positive integer $N$ and a complex parameter $q(0<|q|<1)$. Let $a_{1}, \ldots, a_{N+1}, b_{1}, \ldots, b_{N+1}, c_{1}, c_{2}, d_{1}, d_{2} \in \boldsymbol{C}^{\times}$be complex parameters with a constraint $\prod_{i=1}^{N+1} a_{i} / b_{i}=q \prod_{i=1}^{2} c_{i} / d_{i}$ and $T_{a}: a \mapsto q a$ be the $q$-shift operator of parameter $a$.

In this section, we put

$$
T:=T_{a_{1}}^{-1} T_{b_{1}}^{-1},
$$

and the corresponding shifts are denoted as $\bar{X}:=T(X)$ and $\underline{X}:=T^{-1}(X)$. The operator $T$ plays the role of time evolution of the $q$-Garnier system. Though one can choose any $a_{i}, b_{j}$ instead of $a_{1}, b_{1}$, we consider the case $(i, j)=(1,1)$ for notational simplicity.

For an unknown function $y(x)$, we consider two linear difference equations: $L_{2}(x)=0$ between $y(x), y(q x), \bar{y}(x)$ and $L_{3}(x)=0$ between $y(x), \bar{y}(x)$, $\bar{y}(x / q)$ defined as follows:

$$
\begin{aligned}
& L_{2}(x):=F(f, x) \bar{y}(x)-A_{1}(x) y(q x)+\left(x-b_{1}\right) G(g, x) y(x), \\
& L_{3}(x):=F\left(\bar{f}, \frac{x}{q}\right) y(x)+\left(x-a_{1}\right) G\left(g, \frac{x}{q}\right) \bar{y}(x)-q c_{1} c_{2} B_{1}\left(\frac{x}{q}\right) \bar{y}\left(\frac{x}{q}\right),
\end{aligned}
$$


where

$$
\begin{aligned}
& A(x)=\prod_{i=1}^{N+1}\left(x-a_{i}\right), \quad B(x)=\prod_{i=1}^{N+1}\left(x-b_{i}\right), \quad A_{1}(x)=\frac{A(x)}{x-a_{1}}, \\
& B_{1}(x)=\frac{B(x)}{x-b_{1}}, \quad F(f, x)=\sum_{i=0}^{N} f_{i} x^{i}, \quad G(g, x)=\sum_{i=0}^{N-1} g_{i} x^{i},
\end{aligned}
$$

and $\bar{f}_{i}=T\left(f_{i}\right)$, and $f_{0}, \ldots, f_{N}, g_{0}, \ldots, g_{N-1} \in \boldsymbol{P}^{1}$ are variables depending on parameters $a_{i}, b_{i}, c_{i}, d_{i}$.

In this paper, we call $q^{\rho_{0}}\left(\right.$ resp. $\left.q^{\rho_{\infty}}\right)$ the exponents at $x=0($ resp. $x=\infty)$ when solutions $y(x)$ have the form

$$
\begin{gathered}
y(x)=k_{0} x^{\rho_{0}}(1+O(x)) \quad \text { at } x=0, \\
y(x)=k_{\infty} x^{\rho_{\infty}}\left(1+O\left(\frac{1}{x}\right)\right) \quad \text { at } x=\infty .
\end{gathered}
$$

Proposition 2.1. We assume that the exponents of solutions $y(x)$ of the linear equations $L_{2}(x)=0$ and $L_{3}(x)=0(2.2)$ are $q^{\rho_{0}}=d_{1}, d_{2}$ and $q^{\rho_{\infty}}=c_{1}, c_{2}$. Then the compatibility of the equations $L_{2}(x)=0$ and $L_{3}(x)=0$ gives the following conditions:

$$
\begin{gathered}
c_{1} c_{2} A_{1}(x) B_{1}(x)-\left(x-a_{1}\right)\left(x-b_{1}\right) G(g, x) G(\underline{g}, x)=0 \quad \text { for } F(f, x)=0, \\
q c_{1} c_{2} A_{1}(x) B_{1}(x)-F(f, x) F(\bar{f}, x)=0 \quad \text { for } G(g, x)=0, \\
f_{N} \bar{f}_{N}=q\left(g_{N-1}-c_{1}\right)\left(g_{N-1}-c_{2}\right), \quad f_{0} \bar{f}_{0}=a_{1} b_{1}\left(g_{0}-e_{1}\right)\left(g_{0}-e_{2}\right),
\end{gathered}
$$

where $e_{i}=d_{i} v / a_{1} b_{1}$ and $v=\prod_{i=1}^{N+1}\left(-a_{i}\right)$.

Proof. Under the condition $F(f, x)=0$, eliminating $y(x)$ and $y(q x)$ from $L_{2}(x)=\underline{L}_{3}(q x)=0$, we obtain eq. (2.6). Similarly, for $G(g, x)=0$, eliminating $y(q x)$ and $\bar{y}(x)$ from $L_{2}(x)=L_{3}(q x)=0$, we have eq. (2.7). Considering exponents of the solutions $y(x)$ around $x=0$ and $x=\infty$, by the expansions (2.4) and (2.5), we obtain eq. (2.8).

Though the conditions (2.6)-(2.8) are given as birational equations for $2 N+1$ variables $f_{0}, \ldots, f_{N}, g_{0}, \ldots, g_{N-1}$, they can be reduced to birational equations for $2 N$ variables $f_{1} / f_{0}, \ldots, f_{N} / f_{0}, g_{0}, \ldots, g_{N-1}$ (see eqs. (2.20)-(2.22) in Section 2.4). In Section 2.3, eqs. (2.6)-(2.8) are proved to be sufficient for the compatibility of the equations $L_{2}(x)=0$ and $L_{3}(x)=0$ (2.2).

The most fundamental object is the linear difference equation $L_{1}(x)=0$ between $y(q x), y(x)$ and $y(x / q)$. Eliminating $\bar{y}(x)$ and $\bar{y}(x / q)$ from $L_{2}(x)=$ 
$L_{2}(x / q)=L_{3}(x)=0(2.2)$, we have the linear equation $L_{1}(x)=0$, where

$$
\begin{aligned}
L_{1}(x):= & A(x) F\left(f, \frac{x}{q}\right) y(q x)+q c_{1} c_{2} B\left(\frac{x}{q}\right) F(f, x) y\left(\frac{x}{q}\right) \\
- & \left\{\left(x-a_{1}\right)\left(x-b_{1}\right) F\left(f, \frac{x}{q}\right) G(g, x)\right. \\
& \left.+\frac{F(f, x)}{G(g, x / q)} V\left(f, \bar{f}, \frac{x}{q}\right)\right\} y(x),
\end{aligned}
$$

and

$$
V(f, \bar{f}, x)=q c_{1} c_{2} A_{1}(x) B_{1}(x)-F(f, x) F(\bar{f}, x) .
$$

Lemma 2.2. The equation $L_{1}(x)=0$ (2.9) has the following properties:

(i) It is a linear three term equation between $y(q x), y(x)$ and $y(x / q)$, and the coefficients of $y(q x), y(x)$ and $y(x / q)$ are polynomials of degree $2 N+1$ in $x$,

(ii) The coefficient of $y(q x)$ (resp. $y(x / q)$ ) has zeros at $x=a_{1}, \ldots, a_{N+1}$ (resp. $x=q b_{1}, \ldots, q b_{N+1}$ ),

(iii) The exponents of solutions $y(x)$ are $d_{1}, d_{2}($ at $x=0)$ and $c_{1}, c_{2}$ (at $x=\infty)$,

(iv) The $N$ points $x$ such that $F(f, x)=0$ are apparent singularities (i.e., the solutions are regular there), where

$$
\frac{y(q x)}{y(x)}=\frac{G(g, x)\left(x-b_{1}\right)}{A_{1}(x)} \quad \text { for } F(f, x)=0
$$

holds.

Conversely, the equation $L_{1}(x)=0(2.9)$ is uniquely characterized by these properties (i)-(iv).

Proof. The properties (i)-(iv) follows by computation using eqs. (2.7) and (2.8). The polynomiality of the coefficient of $y(x)$ follows from eq. (2.7). The converse can easily be confirmed by counting the number of free coefficients.

\subsection{Correspondence to Sakai's Lax form}

In [24], Sakai formulated the $q$-Garnier system as a multivariable extension of the sixth $q$-Painlevé equation, by using the connection preserving deformation of the following linear $q$-difference equation:

$$
Y(q x)=\mathscr{A}(x) Y(x), \quad \mathscr{A}(x):=\left[\begin{array}{ll}
a(x) & b(x) \\
c(x) & d(x)
\end{array}\right], \quad Y(x):=\left[\begin{array}{c}
\tilde{y}_{1}(x) \\
\tilde{y}_{2}(x)
\end{array}\right] .
$$


Let $\alpha_{1}, \ldots \alpha_{2 N+2}, \kappa_{1}, \kappa_{2}, \theta_{1}, \theta_{2} \in \boldsymbol{C}^{\times}$be complex parameters. The coefficient matrix $\mathscr{A}(x)$ are defined by the following conditions: (i) $\mathscr{A}(x)=\sum_{i=0}^{N+1} A_{i} x^{i}$, (ii) $A_{N+1}=\operatorname{diag}\left(\kappa_{1}, \kappa_{2}\right)$ and $A_{0}$ has eigenvalues $\theta_{1}$ and $\theta_{2}$. (iii) $\operatorname{det} \mathscr{A}(x)=$ $\kappa_{1} \kappa_{2} \prod_{i=1}^{2 N+2}\left(x-\alpha_{i}\right)$, such that $\kappa_{1} \kappa_{2} \prod_{i=1}^{2 N+2} \alpha_{i}=\theta_{1} \theta_{2}$. The conditions (i)-(iii) determine the matrix $\mathscr{A}(x)$ up to $2 N+1$ free parameters. $2 N$ of them are the dependent variables of the $q$-Garnier system, and one natural choice of them are given by variables $\left\{\lambda_{i}, \tilde{\mu}_{i}\right\}_{i=1}^{N} \in \boldsymbol{P}^{1} \times \boldsymbol{P}^{1}$ where $b\left(\lambda_{i}\right)=0$ and $\tilde{\mu}_{i}=a\left(\lambda_{i}\right)=$ $\tilde{y}_{1}\left(q \lambda_{i}\right) / \tilde{y}_{1}\left(\lambda_{i}\right)$ (a kind of Sklyanin's "magic recipe", see [27, 28] for example). We note that $\mu_{i}$ in [24] is rewritten as $\tilde{\mu}_{i}$ here since $\mu_{i}$ is used for another variable (see Section 2.4). The remaining one free parameter (the normalization of the polynomial $b(x))$ is a gauge parameter.

The system (2.12) can be equivalently described by the following scalar equation for the first component $\tilde{y}_{1}(x)$ :

$$
\begin{gathered}
b\left(\frac{x}{q}\right) \tilde{y}_{1}(q x)-\left\{b\left(\frac{x}{q}\right) a(x)+b(x) d\left(\frac{x}{q}\right)\right\} \tilde{y}_{1}(x) \\
+b(x) \operatorname{det} \mathscr{A}\left(\frac{x}{q}\right) \tilde{y}_{1}\left(\frac{x}{q}\right)=0 .
\end{gathered}
$$

Here $N$ points such that $b(x)=0$ (i.e., $x=\lambda_{i}$ ) are apparent singularities.

Proposition 2.3. The linear equation $L_{1}(x)=0(2.9)$ is equivalent to the linear equation (2.13) up to a gauge transformation and changes of variables and parameters.

Proof. By a gauge transformation: $\quad \tilde{y}_{1}(x)=H(x) y_{1}(x)$ with $H(q x) / H(x)$ $=\prod_{i=1}^{N+1}\left(x-\alpha_{i}\right)$, the system $(2.13)$ can be written as

$$
\begin{aligned}
& \prod_{i=1}^{N+1}\left(x-\alpha_{i}\right) b\left(\frac{x}{q}\right) y_{1}(q x)-\left\{b\left(\frac{x}{q}\right) a(x)+b(x) d\left(\frac{x}{q}\right)\right\} y_{1}(x) \\
& +\kappa_{1} \kappa_{2} \prod_{i=N+2}^{2 N+2}\left(\frac{x}{q}-\alpha_{i}\right) b(x) y_{1}\left(\frac{x}{q}\right)=0 .
\end{aligned}
$$

Then, eq. (2.14) has the following properties:

(i) It is a linear three term equation between $y_{1}(q x), y_{1}(x)$ and $y_{1}(x / q)$, and the coefficients of $y_{1}(q x), y_{1}(x)$ and $y_{1}(x / q)$ are polynomials of degree $2 N+1$ in $x$,

(ii) The coefficient of $y_{1}(q x)$ (resp. $y_{1}(x / q)$ ) has zeros at $x=\alpha_{1}, \ldots, \alpha_{N+1}$ (resp. $x=q \alpha_{N+2}, \ldots, q \alpha_{2 N+2}$ ).

(iii) The exponents of the solutions $y_{1}(x)$ are $\theta_{1} / \prod_{i=1}^{N+1}\left(-\alpha_{i}\right)$, $\theta_{2} / \prod_{i=1}^{N+1}\left(-\alpha_{i}\right)($ at $x=0)$ and $\kappa_{1}, q^{-1} \kappa_{2}($ at $x=\infty)$ 
(iv) $N$ points $x=\lambda_{i}$ such that $b(x)=0$ are apparent singularities, where

$$
\frac{y_{1}\left(q \lambda_{i}\right)}{y_{1}\left(\lambda_{i}\right)}=\frac{\tilde{\mu}_{i}}{\prod_{i=1}^{N+1}\left(\lambda_{i}-\alpha_{i}\right)}
$$

holds.

Conversely, eq. (2.14) is uniquely characterized by these properties (i)-(iv). Hence we see that the equation $L_{1}(x)=0$ (2.9) is equivalent to eq. (2.14) up to changes of variables and parameters due to Lemma 2.2.

\subsection{Sufficiency for the compatibility}

Here we show that the birational equations (2.6)-(2.8) are sufficient for the compatibility of the equations $L_{1}(x)=0(2.9)$ and $L_{2}(x)=0$ (or $L_{3}(x)=0$ ) (2.2). Hence eqs. (2.6)-(2.8) can be regarded as the $q$-Garnier system.

To prove the sufficiency, we first study the linear difference equation $L_{1}^{*}(x)=0$ between $\bar{y}(q x), \bar{y}(x)$ and $\bar{y}(x / q)$. Eliminating $y(x)$ and $y(q x)$ from $L_{2}(x)=L_{3}(x)=L_{3}(q x)=0$ (2.2), we have the following expression:

$$
\begin{gathered}
L_{1}^{*}(x):=\bar{A}(x) F\left(\bar{f}, \frac{x}{q}\right) \bar{y}(q x)+q c_{1} c_{2} \bar{B}\left(\frac{x}{q}\right) F(\bar{f}, x) \bar{y}\left(\frac{x}{q}\right) \\
-\frac{1}{q}\left\{\left(x-a_{1}\right)\left(x-b_{1}\right) F(\bar{f}, x) G\left(g, \frac{x}{q}\right)\right. \\
\left.+\frac{F(\bar{f}, x / q)}{G(g, x)} V(f, \bar{f}, x)\right\} \bar{y}(x),
\end{gathered}
$$

where $V(f, \bar{f}, x)$ is given in eq. (2.10).

The following can be proved in the similar way as Lemma 2.2.

Lemma 2.4. The equation $L_{1}^{*}(x)=0$ (2.16) has the following properties:

(i) It is a linear three term equation between $\bar{y}(q x), \bar{y}(x)$ and $\bar{y}(x / q)$, and the coefficients of $\bar{y}(q x), \bar{y}(x)$ and $\bar{y}(x / q)$ are polynomials of degree $2 N+1$ in $x$,

(ii) The coefficient of $\bar{y}(q x)$ (resp. $\bar{y}(x / q)$ ) has zeros at $x=a_{1} / q, a_{2}, \ldots$, $a_{N+1}$ (resp. $\left.x=b_{1}, q b_{2}, \ldots, q b_{N+1}\right)$,

(iii) The exponents of solutions $\bar{y}(x)$ are $d_{1}, d_{2}($ at $x=0)$ and $c_{1}, c_{2}$ (at $x=\infty)$,

(iv) The $N$ points $x$ such that $F(\bar{f}, x)=0$ are apparent singularities, where

$$
\frac{\bar{y}(q x)}{\bar{y}(x)}=\frac{B_{1}(x)}{c_{1} c_{2} G(g, x)\left(x-a_{1} / q\right)} \quad \text { for } F(\bar{f}, x)=0
$$

holds.

Conversely, the equation $L_{1}^{*}(x)=0(2.16)$ is uniquely characterized by these properties (i)-(iv). 
Theorem 2.5. The linear difference equations $L_{1}(x)=0(2.9)$ and $L_{2}(x)=0$ (2.2) are compatible if and only if the birational equations (2.6)-(2.8) for variables $f_{0}, \ldots, f_{N}$ and $g_{0}, \ldots, g_{N-1}$ are satisfied.

Proof. The compatibility means that $T\left(L_{1}\right)=L_{1}^{*}$, i.e. the commutativity of the following:

$$
\begin{array}{ccc}
L_{1}(\text { Lemma 2.2) } & = & L_{1}(2.9) \\
\downarrow & \uparrow & \uparrow \text {-shift } \\
& & L_{2}, L_{3}(2.2) \\
& & \downarrow \\
L_{1}^{*}(\text { Lemma 2.4 }) & = & L_{1}^{*}(2.16) .
\end{array}
$$

This can be checked by the characterizations of the equations $L_{1}=0$ and $L_{1}^{*}=0$ in Lemmas 2.2 and 2.4 , and the relation: $T(2.11)=(2.17)$ which follows from eq. (2.6).

\subsection{Expressions in terms of roots}

Introduce variables $\lambda_{i}$ and $\mu_{i}(i=1, \ldots, N) \in \boldsymbol{P}^{1}$ such that $F(f, x)=$ $f_{N} \prod_{i=1}^{N}\left(x-\lambda_{i}\right)=: \Lambda(x)$ and $\mu_{i}:=y\left(q \lambda_{i}\right) / y\left(\lambda_{i}\right)$. By the definition of $\mu_{i}$ and eq. (2.11), the variables $\left\{\mu_{i}\right\}$ are related to $\left\{g_{i}\right\}$ as $\mu_{i}=\left(\lambda_{i}-b_{1}\right) G\left(g, \lambda_{i}\right) / A_{1}\left(\lambda_{i}\right)$. Using the characterization in Lemma 2.2 and the partial fraction expansion, one can rewrite linear three term equation $L_{1}(x)=0(2.9)$ as

$$
\begin{aligned}
\frac{L_{1}(x)}{x \Lambda(x) \Lambda(x / q)}= & \frac{A(x)}{x \Lambda(x)} y(q x)+\frac{q c_{1} c_{2} B(x / q)}{x \Lambda(x / q)} y\left(\frac{x}{q}\right) \\
- & {\left[\frac{v\left(d_{1}+d_{2}\right)}{f_{0} x}+\frac{c_{1}+c_{2}}{f_{N}}\right.} \\
& \left.+\sum_{i=1}^{N} \frac{1}{\lambda_{i} \Lambda^{\prime}\left(\lambda_{i}\right)}\left(\frac{A\left(\lambda_{i}\right) \mu_{i}}{x-\lambda_{i}}+\frac{q c_{1} c_{2} B\left(\lambda_{i}\right)}{\left(x-q \lambda_{i}\right) \mu_{i}}\right)\right] y(x)=0,
\end{aligned}
$$

where $f_{0}=f_{N} \prod_{i=1}^{N}\left(-\lambda_{i}\right)$ and $v=\prod_{i=1}^{N+1}\left(-a_{i}\right)$.

Remark 2.6 (On the characterization in terms of variables $\left(\lambda_{i}, \mu_{i}\right)$ ). The characterization in Lemma 2.2 is that for the polynomial in $x$, however we have a similar characterization as a curve in the coordinates $\left(\lambda_{i}, \mu_{i}\right)$. Namely, in case of $i=1$ for example, the equation $x \Lambda(x) \Lambda(x / q) L_{1}=0$ has the following characterization in $\left(\lambda_{1}, \mu_{1}\right)$ : (i) It is a polynomial equation of bidegree $(N+2,2)$, (ii) passing through the following $3 N+9$ points $\left(0, d_{i}\right)_{i=1}^{2},\left(\infty, c_{i}\right)_{i=1}^{2},\left(b_{i}, 0\right)_{i=1}^{N+1}$, $\left(a_{i}, \infty\right)_{i=1}^{N+1}, \quad(x, 0), \quad(x / q, \infty), \quad(x, y(q x) / y(x)), \quad(x / q, y(x) / y(x / q)), \quad\left(\lambda_{i}, \mu_{i}\right)_{i=2}^{N}$ 
$(i \neq 1)$. This characterization is a generalization of those of Lax equations $L_{1}$ for the discrete Painlevé equations as a curve of bidegree $(3,2)$ passing through 12 points [10, 33, 34].

The linear three term equations $L_{2}(x)=0$ and $L_{3}(x)=0(2.2)$ can also be rewritten as

$$
\begin{aligned}
& L_{2}(x)=\Lambda(x) \bar{y}(x)-A_{1}(x) y(q x)+\left(x-b_{1}\right) \Xi(x) y(x)=0, \\
& L_{3}(x)=\bar{\Lambda}\left(\frac{x}{q}\right) y(x)+\left(x-a_{1}\right) \Xi\left(\frac{x}{q}\right) \bar{y}(x)-q c_{1} c_{2} B_{1}\left(\frac{x}{q}\right) \bar{y}\left(\frac{x}{q}\right)=0,
\end{aligned}
$$

where $\Xi(x)=G(g, x)=g_{N-1} \prod_{i=1}^{N-1}\left(x-\xi_{i}\right)$ with the roots $\xi_{i}$ of the polynomial $\Xi(x)$. Then the evolution equation (2.6)-(2.8) can be rewritten as

$$
\begin{gathered}
\Xi\left(\lambda_{i}\right) \underline{\Xi}\left(\lambda_{i}\right)=c_{1} c_{2} \frac{A_{1}\left(\lambda_{i}\right) B_{1}\left(\lambda_{i}\right)}{\left(\lambda_{i}-a_{1}\right)\left(\lambda_{i}-b_{1}\right)} \quad(i=1, \ldots, N), \\
\frac{\Lambda\left(\xi_{i}\right) \bar{\Lambda}\left(\xi_{i}\right)}{f_{N} \bar{f}_{N}}=c_{1} c_{2} \frac{A_{1}\left(\xi_{i}\right) B_{1}\left(\xi_{i}\right)}{\left(g_{N-1}-c_{1}\right)\left(g_{N-1}-c_{2}\right)} \quad(i=1, \ldots, N-1), \\
\prod_{i=1}^{N} \lambda_{i} \bar{\lambda}_{i}=\frac{a_{1} b_{1}}{q} \frac{\left(g_{0}-e_{1}\right)\left(g_{0}-e_{2}\right)}{\left(g_{N-1}-c_{1}\right)\left(g_{N-1}-c_{2}\right)},
\end{gathered}
$$

where $g_{0}=g_{N-1} \prod_{i=1}^{N-1}\left(-\xi_{i}\right)$. The evolution equation $(2.20)-(2.22)$ is the $q$-Garnier equation in terms of $2 N$ variables $\lambda_{1}, \ldots, \lambda_{N}, \xi_{1}, \ldots, \xi_{N-1}, g_{0}$ (or $\left.g_{N-1}\right)$. It is more explicit than eqs. (2.6)-(2.8) but nonbirational.

\subsection{Reduction to the $q$-Painlevé equations}

We give a few comments on lower cases $N=1,2,3$. In [24], the $q$-Painlevé equation of type $D_{5}^{(1)}$ has appeared as a case for the $q$-Garnier system with $N=1$. This is easily seen from eqs. (2.6)-(2.8). For $N=2$ case, it is known [26] that the $q$-Painlevé equation of type $E_{6}^{(1)}$ appears as a particular case for the $q$-Garnier system with $N=2$. In fact, we have

Proposition $2.7([26])$. For the case $N=2$ with a constraint $c_{1}=c_{2}$, the q-Garnier equation (2.6)-(2.8) admits the following reduction

$$
\begin{aligned}
& (f g-1)(f \underline{g}-1)=\frac{\left(1-a_{2} f\right)\left(1-a_{3} f\right)\left(1-b_{2} f\right)\left(1-b_{3} f\right)}{\left(1-a_{1} f\right)\left(1-b_{1} f\right)} \\
& (f g-1)(f \bar{g}-1)=\frac{\left(1-g / a_{2}\right)\left(1-g / a_{3}\right)\left(1-g / b_{2}\right)\left(1-g / b_{3}\right)}{\left(1-c_{1} g / e_{1}\right)\left(1-c_{1} g / e_{2}\right)},
\end{aligned}
$$

where $e_{i}=d_{i} v / a_{1} b_{1}$ and $v=\prod_{i=1}^{N+1}\left(-a_{i}\right)$. 
Proof. Under the case $N=2$ with the constraint, eqs. (2.6)-(2.8) admit a specialization $f_{2}=0$ and $g_{1}=c_{1}$. Then we obtain the results where $f=-f_{1} / f_{0}$ and $g=-g_{0} / c_{1}$.

Eq. (2.23) is the $q$-Painlevé equation of type $E_{6}^{(1)}[10,23]$.

For $N=3$ case, the $q$-Painlevé equation of type $E_{7}^{(1)}$ appears as a particular case for the $q$-Garnier system with $N=3$. In fact, we have

Proposition 2.8. For the case $N=3$ with constraints $d_{1}=d_{2}$ and $c_{1}=c_{2}$, the q-Garnier equation (2.6)-(2.8) admits the following reduction

$$
\begin{aligned}
& \left\{g+\left(f+\frac{e_{1}}{c_{1} f}\right)\right\}\left\{\underline{g}+\left(f+\frac{q e_{1}}{c_{1} f}\right)\right\} \\
& \quad=\frac{\left(f-a_{2}\right)\left(f-a_{3}\right)\left(f-a_{4}\right)\left(f-b_{2}\right)\left(f-b_{3}\right)\left(f-b_{4}\right)}{f^{2}\left(f-a_{1}\right)\left(f-b_{1}\right)}, \\
& \frac{\left(1-x_{1} / f\right)\left(1-x_{1} / \bar{f}\right)}{\left(1-x_{2} / f\right)\left(1-x_{2} / \bar{f}\right)} \\
& \quad=\frac{x_{2}^{2}\left(x_{1}-a_{2}\right)\left(x_{1}-a_{3}\right)\left(x_{1}-a_{4}\right)\left(x_{1}-b_{2}\right)\left(x_{1}-b_{3}\right)\left(x_{1}-b_{4}\right)}{x_{1}^{2}\left(x_{2}-a_{2}\right)\left(x_{2}-a_{3}\right)\left(x_{2}-a_{4}\right)\left(x_{2}-b_{2}\right)\left(x_{2}-b_{3}\right)\left(x_{2}-b_{4}\right)},
\end{aligned}
$$

where $e_{i}=d_{i} v / a_{1} b_{1}, v=\prod_{i=1}^{N+1}\left(-a_{i}\right)$ and $x=x_{1}, x_{2}$ are solutions of the equation $g+\left(x+e_{1} / c_{1} x\right)=0$.

Proof. Under the case $N=3$ with the constraints, eqs. (2.6)-(2.8) admit a specialization $f_{0}=f_{3}=0, g_{0}=e_{1}$ and $g_{2}=c_{1}$. Then we obtain the results where $f=-f_{1} / f_{2}$ and $g=g_{1} / c_{1}$.

Eq. (2.25) for the variables $f$ and $g$ is a kind of the $q$-Painlevé equation of type $E_{7}^{(1)}$, but the direction of the time evolution is different from the standard one given in $[10,23]$. The relation of them will be discussed in $[18,20]$.

\subsection{Correspondence of parameters and variables in $\S 2, \S 4, \S 5$}

In this paper, parameters $a_{1}, \ldots, a_{N+1}, b_{1}, \ldots, b_{N+1}, c_{1}, c_{2}, d_{1}, d_{2}, m, n$ and variables $f_{0}, \ldots, f_{N}, g_{0}, \ldots, g_{N-1}$ are used in slightly different means in $\S 2, \S 4$ and $\S 5$. Their relations are given as follows:

$$
\begin{aligned}
& \left(a_{i}, b_{i}\right)^{\S 2}=\left(\frac{1}{a_{i}}, \frac{1}{b_{i}}\right)^{\S 4}=\left(\frac{1}{a_{i}}, \frac{1}{b_{i}}\right)^{\S 5} \quad(i=1, \ldots, N+1), \\
& \left(a_{N+1}, b_{N+1}\right)^{\S 2}=\left(\frac{1}{a_{N+1}}, \frac{1}{b_{N+1}}\right)^{\S 4}=\left(q^{m+n}, \frac{1}{q}\right)^{\S 5},
\end{aligned}
$$




$$
\begin{aligned}
\left(c_{1}, c_{2}\right)^{\S 2}=\left(q^{m}, q^{n} \prod_{j=1}^{N+1} \frac{b_{j}}{a_{j}}\right)^{\S 4}=\left(q^{m}, c q^{n} \prod_{j=1}^{N} \frac{b_{j}}{a_{j}}\right)^{\S 5}, \\
\left(d_{1}, d_{2}\right)^{\S 2}=\left(1, q^{m+n+1}\right)^{\S 4}=(1, c)^{\S 5}, \\
f_{0}^{\S 2}=\left(\frac{-a_{1}}{v}\left(1-g_{0}\right)\right)^{\S 4}=\left(\frac{-a_{1}}{v}\left(1-g_{0}\right)\right)^{\S 5}, \\
f_{i}^{\S 2}=\left(\frac{-a_{1}}{v}\left(1-g_{0}\right) f_{i}\right)^{\S 4}=\left(\frac{-a_{1}}{v}\left(1-g_{0}\right) f_{i}\right)^{\S 5} \quad(i=1, \ldots, N), \\
\bar{f}_{0}^{\S 2}=\left(\frac{-b_{1} q^{m+n+1}}{v}\left(1-\frac{g_{0}}{q^{m+n+1}}\right)\right)^{\S 4}=\left(\frac{-b_{1} c}{v}\left(1-\frac{g_{0}}{c}\right)\right)^{\S 5}, \\
\bar{f}_{i}^{\S 2}=\left(\frac{-b_{1} q^{m+n+1}}{v}\left(1-\frac{g_{0}}{q^{m+n+1}}\right) \bar{f}_{i}\right)^{\S 4} \\
=\left(\frac{-b_{1} c}{v}\left(1-\frac{g_{0}}{c}\right) \bar{f}_{i}\right)^{\S 5} \quad(i=1, \ldots, N), \\
g_{i}^{\S 2}=\left(\frac{a_{1} b_{1}}{v} g_{i}\right)^{\S 4}=\left(\frac{a_{1} b_{1}}{v} g_{i}\right)^{\S 5} \quad(i=0, \ldots, N-1),
\end{aligned}
$$

where $v=\prod_{i=1}^{N+1}\left(-a_{i}\right)$.

\section{Autonomous case}

In this section, we define a generalization of the QRT system [22] (see also [30]) for hyperelliptic curves and discuss its relation to the $q$-Garnier system.

\subsection{Generalization of the QRT map for hyperelliptic curve}

Let $C$ be a curve of bidegree $(N+1,2)$ on $\boldsymbol{P}^{1} \times \boldsymbol{P}^{1}$ which passes through given $2 N+5$ points $P_{1}, \ldots, P_{2 N+5}$. The number of free parameters of the defining polynomial is $3(N+2)-(2 N+5)=N+1$, hence the curve $C$ forms an $N$ dimensional family, generically of genus $N$.

The dynamical variables of the generalized QRT mapping is a set (a divisor) of $N$ points $\left\{Q_{1}, \ldots, Q_{N}\right\}$ on the curve $C$. Following Mumford [14] we represent it by a pair of functions $\Phi(x)$ and $\Psi(x)$, where $\Phi(x)$ is a polynomial of degree $N$ and $\Psi(x):=S(x) / R(x)$ is a rational function of degree $N$, such that $Q_{i}=\left(x_{i}, \Psi\left(x_{i}\right)\right), \Phi\left(x_{i}\right)=0,(i=1, \ldots, N)$. Note that the normalization of $\Phi(x)$ is irrelevant. A generalized QRT map is defined as follows: 
(1) Fix $N$ free parameters of the curve $C$ so that it passes the initial points $Q_{1}, \ldots, Q_{N}$. We represent the resulting curve as $C_{0}: \varphi(x, y):=\alpha(x) y^{2}+$ $\beta(x) y+\gamma(x)=0$.

(2) Take a subset of indices $I \subset\{1, \ldots, 2 N+5\}$ with $|I|=N+1$ and determine the rational function $\Psi(x)=S(x) / R(x)$ uniquely by the condition that the curve $y=\Psi(x)$ passes through the points $P_{i}=\left(x_{P_{i}}, y_{P_{i}}\right) \quad(i \in I)$ and $Q_{1}, \ldots, Q_{N}$. By definition $R(x)^{2} \varphi(x, \Psi(x))$ is divisible by $\prod_{i \in I}\left(x-x_{P_{i}}\right) \Phi(x)$ and we can define an involution $l_{x}^{I}:(\Phi, \Psi) \mapsto(\tilde{\Phi}, \Psi)$ by the relation

$$
R(x)^{2} \varphi(x, \Psi(x))=\prod_{i \in I}\left(x-x_{P_{i}}\right) \Phi(x) \tilde{\Phi}(x) .
$$

(3) Since the polynomial $\varphi(x, y)$ is of degree 2 in $y$, the other involution $l_{y}:(x, y) \mapsto(x, \tilde{y})$ can be defined simply as $y \tilde{y}=\gamma(x) / \alpha(x)$. Namely we have $\iota_{y}:(\Phi, \Psi) \mapsto(\Phi, \tilde{\Psi})$ where

$$
\Psi(x) \tilde{\Psi}(x) \alpha(x)=\gamma(x) \quad \text { for } \Phi(x)=0 .
$$

(4) We have the generalized QRT map defined by the iteration $T_{I}:=l_{y} l_{x}^{I}$ or $T_{I}^{-1}:=l_{x}^{I} l_{y}$. They are commutative: $T_{I} T_{J}=T_{J} T_{I}$ since they are translations on the Jacobian of the curve $C_{0}$.

\subsection{Relation to the $q$-Garnier system}

In order to apply the algorithm in Section 3.1 to the $q$-Garnier system (2.6)-(2.8), we consider the following case. The points $P_{1}, \ldots, P_{2 N+5}$ are taken as intersection points of the curve $C_{0}$ and four lines ${ }^{1}$ : $x=0, x=\infty, y=0$ and $y=\infty$. Then we have one additional point $P_{2 N+6}$ and the $2 N+6$ points can be parametrized as $\left(a_{i}, \infty\right)_{i=1}^{N+1},\left(b_{i}, 0\right)_{i=1}^{N+1},\left(\infty, c_{i}\right)_{i=1}^{2}$ and $\left(0, d_{i}\right)_{i=1}^{2}$ with a constraint $\prod_{i=1}^{N+1} a_{i} / b_{i}=\prod_{i=1}^{2} c_{i} / d_{i}$. Therefore the curve $C_{0}: \varphi(x, y)=0$ of bidegree $(N+1,2)$ can be written as

$$
\begin{aligned}
& \varphi(x, y):=A(x) y^{2}-U(x) y+c_{1} c_{2} B(x)=0, \\
& U(x):=v\left(d_{1}+d_{2}\right)+\sum_{i=1}^{N} u_{i} x^{i}+\left(c_{1}+c_{2}\right) x^{N+1} .
\end{aligned}
$$

Here $A(x)=\prod_{i=1}^{N+1}\left(x-a_{i}\right), \quad B(x)=\prod_{i=1}^{N+1}\left(x-b_{i}\right)$ and $v=\prod_{i=1}^{N+1}\left(-a_{i}\right)$ as in Section 2.1. Note that the lowest/highest terms of $\varphi(x, y)$ in $x$ are given by $\left.\varphi\right|_{x^{0}}=v\left(y-d_{1}\right)\left(y-d_{2}\right)$ and $\left.\varphi\right|_{x^{N+1}}=\left(y-c_{1}\right)\left(y-c_{2}\right)$. The parameters (con-

\footnotetext{
1 A similar construction is possible for other curve of bidegree $(2,2)$ instead of the four lines.
} 
served quantities) $u_{1}, \ldots, u_{N}$ are determined by the condition $\varphi\left(Q_{i}\right)=0$ for the initial points: $Q_{1}, \ldots, Q_{N}$.

To adjust the formulation given above to that in Section 2, we take the index set $I$ as $\left\{P_{i} \mid i \in I\right\}=\left\{\left(a_{i}, \infty\right)_{i=2}^{N+1},\left(b_{1}, 0\right)\right\}$, and put $\Phi(x):=F(x)$ and $\Psi(x):=\left(x-b_{1}\right) G(x) / A_{1}(x)$ where $F(x)=\sum_{i=0}^{N} f_{i} x^{i}$ and $G(x)=\sum_{i=0}^{N-1} g_{i} x^{i}$ as in eq. (2.3). Then, the $l_{x}^{I}$-flip defined by eq. (3.1) takes the form

$$
\left(x-a_{1}\right)\left(x-b_{1}\right) G(x)^{2}-U(x) G(x)+c_{1} c_{2} A_{1}(x) B_{1}(x)=F(x) \tilde{F}(x),
$$

where $A_{1}(x)=A(x) /\left(x-a_{1}\right)$ and $B_{1}(x)=B(x) /\left(x-b_{1}\right)$ as in eq. (2.3). This relation determines the polynomial $\tilde{F}(x)$ by

$$
\begin{gathered}
F(x) \tilde{F}(x)=c_{1} c_{2} A_{1}(x) B_{1}(x) \quad \text { for } G(x)=0, \\
f_{N} \tilde{f}_{N}=\left(g_{N-1}-c_{1}\right)\left(g_{N-1}-c_{2}\right), \quad f_{0} \tilde{f}_{0}=a_{1} b_{1}\left(g_{0}-e_{1}\right)\left(g_{0}-e_{2}\right),
\end{gathered}
$$

where $e_{i}=d_{i} v /\left(a_{1} b_{1}\right)$ as in Proposition 2.1. On the other hand, the $l_{y}$-flip (3.2) gives

$$
\left(x-a_{1}\right)\left(x-b_{1}\right) G(x) \tilde{G}(x)=c_{1} c_{2} A_{1}(x) B_{1}(x) \quad \text { for } F(x)=0 .
$$

It is easy to see that

Proposition 3.1. The birational equations (3.5)-(3.7) correspond to the autonomous $(q=1)$ version of the $q$-Garnier system (2.6)-(2.8).

\section{The Padé problem on differential grid}

In this section, we study certain Padé approximation problem and solve it explicitly. As a result, we obtain some special solutions of the $q$-Garnier system given in terms of the $q$-Appell Lauricella function.

\subsection{Lax pair and the $q$-Garnier equation}

In this subsection, starting the Padé approximation problem (4.3), we derive linear difference relations (4.5) and nonlinear relations (4.13)-(4.15).

For complex parameters $a_{i}, \ldots, a_{N+1}, b_{1}, \ldots, b_{N+1} \in C^{\times}$, we put

$$
\psi(x):=\prod_{i=1}^{N+1} \frac{\left(a_{i} x\right)_{\infty}}{\left(b_{i} x\right)_{\infty}} .
$$

Here and in what follows, we use the standard $q$-Pochhammer symbols defined as 


$$
\begin{aligned}
& (z)_{\infty}:=\prod_{i=0}^{\infty}\left(1-q^{i} z\right), \quad(z)_{s}:=\frac{(z)_{\infty}}{\left(z q^{s}\right)_{\infty}}, \\
& \left(z_{1}, z_{2}, \ldots, z_{k}\right)_{s}:=\left(z_{1}\right)_{s}\left(z_{2}\right)_{s} \ldots\left(z_{k}\right)_{s} .
\end{aligned}
$$

Define polynomials $P(x)$ and $Q(x)$ of degree $m$ and $n \in \boldsymbol{Z}_{\geq 0}$ by the following Padé approximation condition:

$$
\psi(x)=\frac{P(x)}{Q(x)}+O\left(x^{m+n+1}\right) .
$$

Here the common normalizations of the polynomials $P(x)$ and $Q(x)$ are fixed as $P(0)=1$ tentatively.

By the change of parameters (2.25), the shift $T$ (2.1) becomes the following form

$$
T=T_{a_{1}} T_{b_{1}} .
$$

Let us consider two linear difference relations: $L_{2}(x)=0$ between $y(x)$, $y(q x), \bar{y}(x)$ and $L_{3}(x)=0$ between $y(x), \bar{y}(x), \bar{y}(x / q)$ satisfied by the functions $y(x)=P(x)$ and $y(x)=\psi(x) Q(x)$. The following proposition shows that these relations are regarded as the Lax equations $L_{2}(x)=0$ and $L_{3}(x)=0$ for the $q$-Garnier system (cf. eq. (2.2)).

Proposition 4.1. The linear relations $L_{2}(x)=0$ and $L_{3}(x)=0$ can be written as follows:

$$
\begin{aligned}
L_{2}(x)= & \left(g_{0}\right)_{1} F(f, x) \bar{y}(x)-A_{1}(x) y(q x)+\left(b_{1} x\right)_{1} G(g, x) y(x)=0, \\
L_{3}(x)= & \left(\frac{g_{0}}{q^{m+n+1}}\right)_{1} F\left(\bar{f}, \frac{x}{q}\right) y(x)+\frac{1}{q^{m+n+1}}\left(a_{1} x\right)_{1} G\left(g, \frac{x}{q}\right) \bar{y}(x) \\
& -B_{1}\left(\frac{x}{q}\right) \bar{y}\left(\frac{x}{q}\right)=0,
\end{aligned}
$$

where

$$
\begin{array}{ll}
A(x)=\prod_{i=1}^{N+1}\left(a_{i} x\right)_{1}, & B(x)=\prod_{i=1}^{N+1}\left(b_{i} x\right)_{1}, \quad F(f, x)=1+\sum_{i=1}^{N} f_{i} x^{i}, \\
A_{1}(x)=\frac{A(x)}{\left(a_{1} x\right)_{1}}, & B_{1}(x)=\frac{B(x)}{\left(b_{1} x\right)_{1}},
\end{array}
$$

Here $\bar{f}_{i}=T\left(f_{i}\right)$, and $f_{1}, \ldots, f_{N}, g_{0}, \ldots, g_{N-1} \in \boldsymbol{P}^{1}$ are some constants depending on parameters $a_{i}, b_{i}, m, n$. 
Proof. By the definition of the linear relations $L_{2}(x)=0$ and $L_{3}(x)=0$, they can be written as

$$
\begin{array}{r}
L_{2}(x) \propto\left|\begin{array}{ccc}
y(x) & y(q x) & \bar{y}(x) \\
P(x) & P(q x) & \bar{P}(x) \\
\psi(x) Q(x) & \psi(q x) Q(q x) & \bar{\psi}(x) \bar{Q}(x)
\end{array}\right|=0, \\
L_{3}(x) \propto\left|\begin{array}{ccc}
y(x) & \bar{y}(x) & \bar{y}\left(\frac{x}{q}\right) \\
P(x) & \bar{P}(x) & \bar{P}\left(\frac{x}{q}\right) \\
\psi(x) Q(x) & \bar{\psi}(x) \bar{Q}(x) & \bar{\psi}\left(\frac{x}{q}\right) \bar{Q}\left(\frac{x}{q}\right)
\end{array}\right|=0 .
\end{array}
$$

Setting

$$
\mathbf{y}(x):=\left[\begin{array}{c}
P(x) \\
\psi(x) Q(x)
\end{array}\right]
$$

define Casorati determinants $D_{1}(x), D_{2}(x)$ and $D_{3}(x)$ by

$$
\begin{aligned}
& D_{1}(x):=\operatorname{det}[\mathbf{y}(x), \mathbf{y}(q x)], \quad D_{2}(x):=\operatorname{det}[\mathbf{y}(x), \overline{\mathbf{y}}(x)], \\
& D_{3}(x):=\operatorname{det}[\mathbf{y}(q x), \overline{\mathbf{y}}(x)] .
\end{aligned}
$$

Then, the linear relations $L_{2}(x)=0$ and $L_{3}(x)=0$ take the following forms:

$$
\begin{aligned}
& L_{2}(x) \propto D_{1}(x) \bar{y}(x)-D_{2}(x) y(q x)+D_{3}(x) y(x)=0, \\
& L_{3}(x) \propto \bar{D}_{1}\left(\frac{x}{q}\right) y(x)+D_{3}\left(\frac{x}{q}\right) \bar{y}(x)-D_{2}(x) \bar{y}\left(\frac{x}{q}\right)=0 .
\end{aligned}
$$

The determinants (4.9) can be computed by the condition (4.3) and the relations

$$
\frac{\psi(q x)}{\psi(x)}=\prod_{i=1}^{N+1} \frac{\left(b_{i} x\right)_{1}}{\left(a_{i} x\right)_{1}}, \quad \frac{\bar{\psi}(x)}{\psi(x)}=\frac{\left(b_{1} x\right)_{1}}{\left(a_{1} x\right)_{1}} .
$$

The results are given as

$$
\begin{aligned}
D_{1}(x) & =\frac{\psi(x)}{A(x)}\{B(x) P(x) Q(q x)-A(x) P(q x) Q(x)\} \\
& =: w_{0} \frac{\psi(x) x^{m+n+1}}{A(x)} F(f, x) \\
D_{2}(x) & =\frac{\psi(x)}{\left(a_{1} x\right)_{1}}\left\{\left(b_{1} x\right)_{1} P(x) \bar{Q}(x)-\left(a_{1} x\right)_{1} \bar{P}(x) Q(x)\right\}=: w_{1} \frac{\psi(x) x^{m+n+1}}{\left(a_{1} x\right)_{1}},
\end{aligned}
$$




$$
\begin{aligned}
D_{3}(x) & =\frac{\psi(x)}{A(x)}\left\{\left(b_{1} x\right)_{1} A_{1}(x) P(q x) \bar{Q}(x)-B(x) \bar{P}(x) Q(q x)\right\} \\
& =: w_{1} \frac{\psi(x) x^{m+n+1}}{A(x)}\left(b_{1} x\right)_{1} G(g, x),
\end{aligned}
$$

with some constants $w_{0}$ and $w_{1}$ depending on parameters $a_{i}, b_{i}, m$ and $n$. The constants $w_{0}$ and $w_{1}$ are fixed as $w_{0}=\left(g_{0}\right)_{1}$ and $w_{1}=\left(g_{0} / q^{m+n+1}\right)_{1}$ by the condition that eq. (4.10) has a solution such as $y(0)=P(0)=1$.

The following proposition can be proved in the similar way as Proposition 2.1 .

Proposition 4.2. The constants $f_{1}, \ldots, f_{N}$ and $g_{0}, \ldots, g_{N-1}$ satisfy the following relations:

$$
\begin{aligned}
& A_{1}(x) B_{1}(x)-\frac{1}{q^{m+n+1}}\left(a_{1} x, b_{1} x\right)_{1} G(g, x) G(\underline{g}, x)=0 \quad \text { for } F(f, x)=0 \\
& A_{1}(x) B_{1}(x)-\left(g_{0}, \frac{g_{0}}{q^{m+n+1}}\right)_{1} F(f, x) F(\bar{f}, x)=0 \quad \text { for } \quad G(g, x)=0 \\
& \left(g_{0}, \frac{g_{0}}{q^{m+n+1}}\right)_{1} f_{N} \bar{f}_{N}=\left(\frac{a_{1} g_{N-1}}{q^{m+n}}+\frac{\prod_{i=2}^{N+1}\left(-b_{i}\right)}{q^{m}}\right)\left(b_{1} g_{N-1}+q^{m} \prod_{i=2}^{N+1}\left(-a_{i}\right)\right)
\end{aligned}
$$

These relations (4.13)-(4.15) are regarded as the $q$-Garnier system (cf. eqs. (2.6)-(2.8)).

\subsection{Special solutions}

We derive the explicit forms (4.23)-(4.25) of variables $\left\{f_{i}, g_{i}\right\}$ appearing in the Casorati determinants $D_{1}(x)$ and $D_{3}(x)(4.12)$. They are interpreted as the special solutions for the $q$-Garnier system (4.13)-(4.15) due to Proposition 4.2.

Proposition 4.3. For any given function $\psi(x)=\sum_{k=0}^{\infty} p_{k} x^{k}, \quad\left(p_{0}=1\right.$, $\left.p_{i}=0, i<0\right)$, the polynomials $P(x)$ and $Q(x)$ of degree $m$ and $n$ for the approximation condition (4.3) are given by

$$
P(x)=\sum_{i=0}^{m} s_{\left(m^{n}, i\right)} x^{i}, \quad Q(x)=\sum_{i=0}^{n} s_{\left((m+1)^{i}, m^{n-i}\right)}(-x)^{i},
$$

where $s_{\lambda}$ is the Schur function defined by the Jacobi Trudi formula

$$
s_{\left(\lambda_{1}, \ldots, \lambda_{l}\right)}:=\operatorname{det}\left(p_{\lambda_{i}-i+j}\right)_{i, j=1}^{l},
$$

and $m^{n}=(\underbrace{m, m, \ldots, m}_{n})$. 
For the proof, see Section 2 of [32] for example.

Lemma 4.4. The polynomials $P(x)$ and $Q(x)$ in Proposition 4.3 can be expressed in terms of a single determinant as

$$
P(x)=\left.x^{m} s_{\left(m^{n+1}\right)}\right|_{p_{i} \rightarrow \sum_{j=0}^{i} x^{-j} p_{i-j}}, \quad Q(x)=\left.(-x)^{n} s_{\left((m+1)^{n}\right)}\right|_{p_{i} \rightarrow p_{i}-x^{-1} p_{i-1}} .
$$

Proof. Direct computation of the right hand side of eqs. (4.18).

Note that the normalization of the polynomials $P(x)$ and $Q(x)$ in eqs. (4.16) and (4.18) are different from the convention $P(0)=1$ in the approximation condition (4.3). However, this difference does not affect the results in the following Proposition 4.6, since the common normalization factors of $P(x)$ and $Q(x)$ are cancels in eqs. (4.27)-(4.29).

We apply the general results described above to the function $\psi(x)$ in eq. (4.1) which can be written as

$$
\psi(x)=\sum_{k=0}^{\infty} p_{k} x^{k}=\exp \left(\sum_{k=1}^{\infty} \sum_{s=1}^{N+1} \frac{b_{s}^{k}-a_{s}^{k}}{k\left(1-q^{k}\right)} x^{k}\right) .
$$

We note that this kind of the expression (4.19) has already appeared in [31].

By the definition (4.1), it is easy to see the properties for $p_{k}$ as follows:

$$
\begin{aligned}
& T_{a_{s}}^{-1}\left(p_{i}\right)=p_{i}-\frac{1}{q} a_{s} p_{i-1}, \quad T_{b_{s}}\left(p_{i}\right)=p_{i}-b_{s} p_{i-1}, \\
& T_{a_{s}}\left(p_{i}\right)=\sum_{j=0}^{i} a_{s}^{j} p_{i-j}, \quad T_{b_{s}}^{-1}\left(p_{i}\right)=\sum_{j=0}^{i}\left(\frac{b_{s}}{q}\right)^{j} p_{i-j},
\end{aligned}
$$

for $s=1, \ldots, N+1$.

Proposition 4.5. The polynomials $P(x)$ and $Q(x)$ have the following special values:

$$
\begin{array}{ll}
P\left(\frac{1}{a_{s}}\right)=\left(\frac{1}{a_{s}}\right)^{m} T_{a_{s}}\left(\tau_{m, n+1}\right), & Q\left(\frac{q}{a_{s}}\right)=\left(-\frac{q}{a_{s}}\right)^{n} T_{a_{s}}^{-1}\left(\tau_{m+1, n}\right), \\
P\left(\frac{q}{b_{s}}\right)=\left(\frac{q}{b_{s}}\right)^{m} T_{b_{s}}^{-1}\left(\tau_{m, n+1}\right), & Q\left(\frac{1}{b_{s}}\right)=\left(-\frac{1}{b_{s}}\right)^{n} T_{b_{s}}\left(\tau_{m+1, n}\right),
\end{array}
$$

for $s=1, \ldots, N+1$. Here $\tau_{m, n}$ is defined as

$$
\tau_{m, n}=s_{\left(m^{n}\right)}=\operatorname{det}\left(p_{m-i+j}\right)_{i, j=1}^{n} .
$$

Proof. Follows from the properties (4.20) and the formula (4.18). 
Proposition 4.6. The polynomials $F(f, x)$ and $G(g, x)$ are determined by the following special values at $x=1 / a_{i}, 1 / b_{i}$ :

$$
\begin{aligned}
\frac{F\left(f, 1 / a_{i}\right)}{F\left(f, 1 / b_{j}\right)}=\alpha \frac{T_{a_{i}}\left(\tau_{m, n+1}\right) T_{a_{i}}^{-1}\left(\tau_{m+1, n}\right)}{T_{b_{j}}^{-1}\left(\tau_{m, n+1}\right) T_{b_{j}}\left(\tau_{m+1, n}\right)} \quad(i, j=1, \ldots, N+1), \\
G\left(g, \frac{1}{a_{i}}\right)=\beta \frac{T_{a_{i}}\left(\bar{\tau}_{m, n+1}\right) T_{a_{i}}^{-1}\left(\tau_{m+1, n}\right)}{T_{a_{1}}\left(\tau_{m, n+1}\right) T_{a_{1}}^{-1}\left(\bar{\tau}_{m+1, n}\right)} \quad(i=2, \ldots, N+1), \\
G\left(g, \frac{1}{b_{i}}\right)=\gamma \frac{T_{b_{i}}^{-1}\left(\tau_{m, n+1}\right) T_{b_{i}}\left(\bar{\tau}_{m+1, n}\right)}{T_{b_{1}}^{-1}\left(\bar{\tau}_{m, n+1}\right) T_{b_{1}}\left(\tau_{m+1, n}\right)} \quad(i=2, \ldots, N+1),
\end{aligned}
$$

where

$$
\alpha=-q^{n-m} \frac{a_{i}}{b_{j}} \frac{B\left(1 / a_{i}\right)}{A\left(1 / b_{j}\right)}, \quad \beta=-q^{n} \frac{a_{i}}{a_{1}} \frac{B_{1}\left(1 / a_{i}\right)}{\left(b_{1} / a_{1}\right)_{1}}, \quad \gamma=-q^{m} \frac{b_{i}}{b_{1}} \frac{A_{1}\left(1 / b_{i}\right)}{\left(a_{1} / b_{1}\right)_{1}} .
$$

Proof. From the first equation of (4.12), we have

$$
\begin{aligned}
\frac{F\left(f, 1 / a_{i}\right)}{F\left(f, 1 / b_{j}\right)}= & -\left(\frac{a_{i}}{b_{j}}\right)^{m+n+1} \frac{B\left(1 / a_{i}\right)}{A\left(1 / b_{j}\right)} \frac{P\left(1 / a_{i}\right) Q\left(q / a_{i}\right)}{P\left(q / b_{j}\right) Q\left(1 / b_{j}\right)} \\
& (i, j=1, \ldots, N+1) .
\end{aligned}
$$

From the second and third equations of (4.12), we have

$$
\begin{aligned}
& G\left(g, \frac{1}{a_{i}}\right)=-\left(\frac{a_{i}}{a_{1}}\right)^{m+n+1} \frac{B_{1}\left(1 / a_{i}\right)}{\left(b_{1} / a_{1}\right)_{1}} \frac{\bar{P}\left(1 / a_{i}\right) Q\left(q / a_{i}\right)}{P\left(1 / a_{1}\right) \bar{Q}\left(1 / a_{1}\right)} \quad(i=2, \ldots, N+1), \\
& G\left(g, \frac{1}{b_{i}}\right)=-\left(\frac{b_{i}}{b_{1}}\right)^{m+n+1} \frac{A_{1}\left(1 / b_{i}\right)}{\left(a_{1} / b_{1}\right)_{1}} \frac{P\left(q / b_{i}\right) \bar{Q}\left(1 / b_{i}\right)}{\bar{P}\left(1 / b_{1}\right) Q\left(1 / b_{1}\right)} \quad(i=2, \ldots, N+1) .
\end{aligned}
$$

Substituting the special values (4.21) into the expressions (4.27)-(4.29) respectively, we obtain eqs. (4.23)-(4.25).

We remark that the function $p_{k}$ can be written in terms of the $q$-Appell Lauricella function $\varphi_{D}^{(l)}[4]$ as follows:

Proposition 4.7. The function $p_{k}$ can be explicitly written as

$$
\begin{aligned}
& p_{k}= \frac{b_{N+1}^{k}\left(a_{N+1} / b_{N+1}\right)_{k}}{(q)_{k}} \\
& \times \varphi_{D}^{(N)}\left(q^{-k}, \frac{a_{1}}{b_{1}}, \ldots, \frac{a_{N}}{b_{N}}, q^{-k+1} \frac{b_{N+1}}{a_{N+1}} ; q \frac{b_{1}}{a_{N+1}}, \ldots, q \frac{b_{N}}{a_{N+1}}\right), \\
& \varphi_{D}^{(l)}\left(\alpha, \beta_{1}, \ldots, \beta_{l}, \gamma ; z_{1}, \ldots, z_{l}\right):=\sum_{m_{i} \geq 0} \frac{(\alpha)_{|m|}\left(\beta_{1}\right)_{m_{1}} \ldots\left(\beta_{l}\right)_{m_{l}}}{(\gamma)_{|m|}(q)_{m_{1}} \ldots(q)_{m_{l}}} z_{1}^{m_{1}} \ldots z_{l}^{m_{l}},
\end{aligned}
$$

where $|m|=m_{1}+\cdots+m_{l}$. 
Proof. By the definition of $\psi(x)$ (4.1) and the $q$-binomial theorem, we have

$$
\psi(x)=\sum_{m_{i} \geq 0} \frac{\left(a_{1} / b_{1}\right)_{m_{1}} \ldots\left(a_{N+1} / b_{N+1}\right)_{m_{N+1}}}{(q)_{m_{1}} \ldots(q)_{m_{N+1}}} b_{1}^{m_{1}} \ldots b_{N+1}^{m_{N+1}} x^{m_{1}+\cdots+m_{N+1}} .
$$

Note that for $k \geq m_{N+1}$, we have

$$
\frac{\left(a_{N+1} / b_{N+1}\right)_{m_{N+1}}}{(q)_{m_{N+1}}}=\frac{\left(a_{N+1} / b_{N+1}\right)_{k}\left(q^{-k}\right)_{k-m_{N+1}}}{(q)_{k}\left(q^{-k+1}\left(b_{N+1} / a_{N+1}\right)\right)_{k-m_{N+1}}}\left(q \frac{b_{N+1}}{a_{N+1}}\right)^{k-m_{N+1}} .
$$

Substituting eq. (4.33) with $k=|m|+m_{N+1}$ into eq. (4.32), we obtain eq. (4.30).

In [25], a hypergeometric solution of the $q$-Garnier system is given in terms of the $q$-Appell Lauricella function $\varphi_{D}^{(N)}(4.31)$. Our result corresponds to its determinantal generalization in terminating case. For the differential Garnier system, a more general determinant formula applicable also to the transcendental solutions is derived by applying the (Hermite-)Padé approximation [11, 12, 13].

\section{The Padé problem on $q$-grid}

In this section, we study certain Padé interpolation problem and solve it explicitly. As a result, we obtain some special solutions of the $q$-Garnier system given in terms of the generalized $q$-hypergeometric function.

\subsection{Lax pair and the $q$-Garnier system}

In this subsection, starting the Padé interpolation problem (5.2), we derive linear difference relations (5.3) and nonlinear relations (5.7)-(5.9).

For complex parameters $a_{1}, \ldots, a_{N}, b_{1}, \ldots, b_{N}, c \in \boldsymbol{C}^{\times}$, we put

$$
\psi(x):=c^{\log _{q} x} \prod_{i=1}^{N} \frac{\left(a_{i} x, b_{i}\right)_{\infty}}{\left(a_{i}, b_{i} x\right)_{\infty}} .
$$

Define polynomials $P(x)$ and $Q(x)$ of degree $m$ and $n \in \boldsymbol{Z}_{\geq 0}$ by the following Padé interpolation condition:

$$
\psi\left(x_{s}\right)=\frac{P\left(x_{s}\right)}{Q\left(x_{s}\right)} \quad\left(x_{s}=q^{s}, s=0,1, \ldots, m+n\right) .
$$

The common normalizations of the polynomials $P(x)$ and $Q(x)$ are fixed as $P(0)=1$ tentatively. As in Section 4.1, the shift $T$ is given by eq. (4.4). 
Proposition 5.1. For $y(x)=P(x)$ and $y(x)=\psi(x) Q(x)$, we have the following linear relations:

$$
\begin{aligned}
L_{2}(x) & =\left(g_{0}\right)_{1} F(f, x) \bar{y}(x)-\left(\frac{x}{q^{m+n}}\right)_{1} A_{1}(x) y(q x)+\left(b_{1} x\right)_{1} G(g, x) y(x)=0, \\
L_{3}(x) & =\left(\frac{g_{0}}{c}\right)_{1} F\left(\bar{f}, \frac{x}{q}\right) y(x)+\frac{1}{c}\left(a_{1} x\right)_{1} G\left(g, \frac{x}{q}\right) \bar{y}(x)-(x)_{1} B_{1}\left(\frac{x}{q}\right) \bar{y}\left(\frac{x}{q}\right) \\
& =0
\end{aligned}
$$

where

$$
\begin{array}{lll}
A(x)=\prod_{j=1}^{N}\left(a_{j} x\right)_{1}, & B(x)=\prod_{j=1}^{N}\left(b_{j} x\right)_{1}, & F(f, x)=1+\sum_{j=1}^{N} f_{j} x^{j}, \\
A_{1}(x)=\frac{A(x)}{\left(a_{1} x\right)_{1}}, & B_{1}(x)=\frac{B(x)}{\left(b_{1} x\right)_{1}}, & G(g, x)=\sum_{j=0}^{N-1} g_{j} x^{j} .
\end{array}
$$

Here $\bar{f}_{i}=T\left(f_{i}\right)$, and $f_{1}, \ldots, f_{N}, g_{0}, \ldots, g_{N-1} \in \boldsymbol{P}^{1}$ are some constants depending on parameters $a_{i}, b_{i}, c, m, n$.

Proof. The method of the proof is the same as that of Proposition 4.1. By the definition of the linear relations $L_{2}(x)=0$ and $L_{3}(x)=0$, they can be written as the expression (4.7). Define Casorati determinants $D_{1}(x), D_{2}(x)$ and $D_{3}(x)$ by eq. (4.9). Then, the linear relations $L_{2}(x)=0$ and $L_{3}(x)=0$ can take the forms (4.10). The determinants (4.9) can be computed by the condition (5.2) and the relations

$$
\frac{\psi(q x)}{\psi(x)}=c \frac{B(x)}{A(x)}, \quad \frac{\bar{\psi}(x)}{\psi(x)}=\frac{\left(a_{1}, b_{1} x\right)_{1}}{\left(a_{1} x, b_{1}\right)_{1}} .
$$

The results are given as

$$
\begin{aligned}
D_{1}(x) & =\frac{\psi(x)}{A(x)}\{c B(x) P(x) Q(q x)-A(x) P(q x) Q(x)\} \\
& =: w_{0} \frac{\psi(x) \prod_{i=0}^{m+n-1}\left(x / q^{i}\right)_{1}}{A(x)} F(f, x), \\
D_{2}(x) & =\frac{\psi(x)}{\left(a_{1} x, b_{1}\right)_{1}}\left\{\left(a_{1}, b_{1} x\right)_{1} P(x) \bar{Q}(x)-\left(a_{1} x, b_{1}\right)_{1} \bar{P}(x) Q(x)\right\} \\
& =: w_{1} \frac{\psi(x) \prod_{i=0}^{m+n}\left(x / q^{i}\right)_{1}}{\left(a_{1} x, b_{1}\right)_{1}},
\end{aligned}
$$




$$
\begin{aligned}
D_{3}(x) & =\frac{\psi(x)}{A(x)\left(b_{1}\right)_{1}}\left\{\left(a_{1}, b_{1} x\right)_{1} A_{1}(x) P(q x) \bar{Q}(x)-c\left(b_{1}\right)_{1} B(x) \bar{P}(x) Q(q x)\right\} \\
& =: w_{1} \frac{\psi(x) \prod_{i=0}^{m+n-1}\left(x / q^{i}\right)_{1}}{A(x)\left(b_{1}\right)_{1}}\left(b_{1} x\right)_{1} G(g, x)
\end{aligned}
$$

with some constants $w_{0}$ and $w_{1}$ depending on parameters $a_{i}, b_{i}, c, m$ and $n$. The constants $w_{0}$ and $w_{1}$ are fixed as $w_{0}=\left(g_{0}\right)_{1}$ and $w_{1}=\left(g_{0} / c\right)_{1}$ by the condition that eq. (4.10) has a solution such as $y(0)=P(0)=1$.

These relations (5.3) are regarded as the Lax equations $L_{2}(x)=0$ and $L_{3}(x)=0$ for the $q$-Garnier system (cf. eq. (2.2)).

Proposition 5.2. The constants $f_{1}, \ldots, f_{N}$ and $g_{0}, \ldots, g_{N-1}$ satisfy the following relations:

$$
\begin{aligned}
& \left(q x, \frac{x}{q^{m+n}}\right)_{1} A_{1}(x) B_{1}(x)-\frac{1}{c}\left(a_{1} x, b_{1} x\right)_{1} G(g, x) G(\underline{g}, x)=0 \\
& \text { for } F(f, x)=0 \\
& \left(q x, \frac{x}{q^{m+n}}\right)_{1} A_{1}(x) B_{1}(x)-\left(g_{0}, \frac{g_{0}}{c}\right)_{1} F(f, x) F(\bar{f}, x)=0 \\
& \text { for } G(g, x)=0 \\
& \left(g_{0}, \frac{g_{0}}{c}\right)_{1} f_{N} \bar{f}_{N}=\left(\frac{q a_{1}}{c} g_{N-1}-\frac{\prod_{i=2}^{N}\left(-b_{i}\right)}{q^{m-1}}\right)\left(b_{1} g_{N-1}-\frac{\prod_{i=2}^{N}\left(-a_{i}\right)}{q^{n}}\right) .
\end{aligned}
$$

Proof. Similar to the proof of Proposition 2.1.

These relations (5.7)-(5.9) are also regarded as the $q$-Garnier system (cf. eqs. $(2.6)-(2.8))$.

\subsection{Special solutions}

We derive the explicit forms (5.17)-(5.19) of variables $\left\{f_{i}, g_{i}\right\}$ appearing in the Casorati determinants $D_{1}(x)$ and $D_{3}(x)$ (5.6). They are interpreted as the special solutions for the $q$-Garnier system (5.7)-(5.9) due to Proposition 5.2.

Proposition 5.3 ([7] also [15]). For a given sequence $\psi_{s}$, the polynomials $P(x)$ and $Q(x)$ of degree $m$ and $n$ for an interpolation problem

$$
\psi_{s}=\frac{P\left(x_{s}\right)}{Q\left(x_{s}\right)} \quad(s=0,1, \ldots, m+n)
$$

are given by the following determinant expressions: 


$$
\begin{aligned}
& P(x)=F(x) \operatorname{det}\left[\sum_{s=0}^{m+n} u_{s} \frac{x_{s}^{i+j}}{x-x_{s}}\right]_{i, j=0}^{n}, \\
& Q(x)=\operatorname{det}\left[\sum_{s=0}^{m+n} u_{s} x_{s}^{i+j}\left(x-x_{s}\right)\right]_{i, j=0}^{n-1},
\end{aligned}
$$

where $u_{s}=\psi_{s} / F^{\prime}\left(x_{s}\right)$ and $F(x)=\prod_{i=0}^{m+n}\left(x-x_{i}\right)$.

Lemma 5.4 ([15]). In the q-grid case of problem (5.10) (i.e., $x_{s}=q^{s}$ ), the formulae (5.11) take the following form:

$$
\begin{aligned}
& P(x)=\frac{F(x)}{(q)_{m+n}^{n+1}} \operatorname{det}\left[\sum_{s=0}^{m+n} \psi_{s} \frac{\left(q^{-(m+n)}\right)_{s}}{(q)_{s}} \frac{q^{s(i+j+1)}}{x-q^{s}}\right]_{i, j=0}^{n}, \\
& Q(x)=\frac{1}{(q)_{m+n}^{n}} \operatorname{det}\left[\sum_{s=0}^{m+n} \psi_{s} \frac{\left(q^{-(m+n)}\right)_{s}}{(q)_{s}} q^{s(i+j+1)}\left(x-q^{s}\right)\right]_{i, j=0}^{n-1} .
\end{aligned}
$$

Proof. In the derivation of (5.12), we have used the relations

$$
F^{\prime}\left(x_{s}\right)=\frac{(q)_{s}(q)_{m+n}}{q^{s}\left(q^{-(m+n)}\right)_{s}} .
$$

Substituting the value of $F^{\prime}\left(x_{s}\right)$ (5.13) into the formulae (5.11), one obtains the determinant formulae (5.12).

The normalization of the polynomials $P(x)$ and $Q(x)$ in eqs. (5.11) and (5.12) are different from the convention $P(0)=1$ in the interpolation condition (5.2). As in Section 4.2, this difference does not affect the results in the following Proposition 5.6.

Proposition 5.5. The polynomials $P(x)$ and $Q(x)$ defined in Section 5.1 have the following special values:

$$
\begin{aligned}
& P\left(\frac{1}{a_{s}}\right)=\frac{\left(a_{s}\right)_{m+n+1}}{a_{s}^{m}\left(a_{s}\right)_{1}^{n+1}(q)_{m+n}^{n+1}} T_{a_{s}}\left(\tau_{m, n}\right), \\
& Q\left(\frac{q}{a_{s}}\right)=\frac{q^{n}\left(a_{s} / q\right)_{1}^{n}}{a_{s}^{n}(q)_{m+n}^{n}} T_{a_{s}}^{-1}\left(\tau_{m+1, n-1}\right), \\
& P\left(\frac{q}{b_{s}}\right)=\frac{q^{m}\left(b_{s} / q\right)_{m+n+1}}{b_{s}^{m}\left(b_{s} / q\right)_{1}^{n+1}(q)_{m+n}^{n+1}} T_{b_{s}}^{-1}\left(\tau_{m, n}\right), \\
& Q\left(\frac{1}{b_{s}}\right)=\frac{\left(b_{s}\right)_{1}^{n}}{b_{s}^{n}(q)_{m+n}^{n}} T_{b_{s}}\left(\tau_{m+1, n-1}\right),
\end{aligned}
$$


for $s=1, \ldots, N$. Here $\tau_{m, n}$ is defined as

$$
\tau_{m, n}=\operatorname{det}\left[{ }_{N+1} \varphi_{N}\left(\begin{array}{c}
b_{1}, \ldots, b_{N}, q^{-(m+n)} \\
a_{1}, \ldots, a_{N}
\end{array}, c q^{i+j+1}\right)\right]_{i, j=0}^{n},
$$

and the q-HGF (the q-hypergeometric functions [4]) is defined by

$$
{ }_{k} \varphi_{l}\left(\begin{array}{c}
\alpha_{1}, \ldots, \alpha_{k} \\
\beta_{1}, \ldots, \beta_{l}
\end{array}, x\right):=\sum_{s=0}^{\infty} \frac{\left(\alpha_{1}, \ldots, \alpha_{k}\right)_{s}}{\left(\beta_{1}, \ldots, \beta_{l}, q\right)_{s}}\left[(-1)^{s} q^{\left(\begin{array}{c}
s \\
2
\end{array}\right)}\right]^{1+l-k} x^{s},
$$

with $\left(\begin{array}{l}s \\ 2\end{array}\right)=s(s-1) / 2$.

Proof. Follows from the formula (5.12) and the sequence $\psi_{s}=\psi\left(q^{s}\right)=$ $c^{s} \prod_{i=1}^{N}\left(b_{i}\right)_{s} /\left(a_{i}\right)_{s}$.

Proposition 5.6. The polynomials $F(f, x)$ and $G(g, x)$ are determined by the following special values at $x=1 / a_{i}, 1 / b_{i}$ :

$$
\begin{aligned}
\frac{F\left(f, 1 / a_{i}\right)}{F\left(f, 1 / b_{j}\right)} & =\alpha \frac{T_{a_{i}}\left(\tau_{m, n}\right) T_{a_{i}}^{-1}\left(\tau_{m+1, n-1}\right)}{T_{b_{j}}^{-1}\left(\tau_{m, n}\right) T_{b_{j}}\left(\tau_{m+1, n-1}\right)} \quad(i, j=1, \ldots, N), \\
G\left(g, \frac{1}{a_{i}}\right) & =\beta \frac{T_{a_{i}}\left(\bar{\tau}_{m, n}\right) T_{a_{i}}^{-1}\left(\tau_{m+1, n-1}\right)}{T_{a_{1}}\left(\tau_{m, n}\right) T_{a_{1}}^{-1}\left(\bar{\tau}_{m+1, n-1}\right)} \quad(i=2, \ldots, N), \\
G\left(g, \frac{1}{b_{i}}\right) & =\gamma \frac{T_{b_{i}}^{-1}\left(\tau_{m, n}\right) T_{b_{i}}\left(\bar{\tau}_{m+1, n-1}\right)}{T_{b_{1}}^{-1}\left(\bar{\tau}_{m, n}\right) T_{b_{1}}\left(\tau_{m+1, n-1}\right)} \quad(i=2, \ldots, N),
\end{aligned}
$$

where

$$
\begin{aligned}
& \alpha=-c q^{n-m} \frac{\left(a_{i} q^{m+n}\right)_{1}\left(b_{j} / q\right)_{1}^{n}\left(a_{i} / q\right)_{1}^{n}}{\left(a_{i}\right)_{1}^{n+1}\left(b_{j}\right)_{1}^{n}} \frac{B\left(1 / a_{i}\right)}{A\left(1 / b_{j}\right)}, \\
& \beta=c \frac{\left(b_{1}, a_{i} q^{m+n}\right)_{1}\left(a_{i} / q\right)_{1}^{n} B_{1}\left(1 / a_{i}\right)}{a_{1} q^{m}\left(b_{1} / a_{1}\right)_{1}\left(a_{i}\right)_{1}^{n+1}}, \quad \gamma=\frac{\left(a_{1}\right)_{1}\left(b_{i}\right)_{1}^{n} A_{1}\left(1 / b_{i}\right)}{b_{1} q^{n}\left(a_{1} / b_{1}\right)_{1}\left(b_{i} / q\right)_{1}^{n}} .
\end{aligned}
$$

Proof. Taking the ratio $D_{1}\left(1 / a_{i}\right) / D_{1}\left(1 / b_{j}\right)$ (5.6), we have

$$
\begin{aligned}
\frac{F\left(f, 1 / a_{i}\right)}{F\left(f, 1 / b_{j}\right)}=-c & \prod_{s=0}^{m+n-1} \frac{\left(1 / b_{j} q^{s}\right)_{1}}{\left(1 / a_{i} q^{s}\right)_{1}} \frac{B\left(1 / a_{i}\right)}{A\left(1 / b_{j}\right)} \frac{P\left(1 / a_{i}\right) Q\left(q / a_{i}\right)}{P\left(q / b_{j}\right) Q\left(1 / b_{j}\right)} \\
& (i, j=1, \ldots, N) .
\end{aligned}
$$

Taking the ratio $D_{3}\left(1 / a_{i}\right) / D_{2}\left(1 / a_{1}\right)$ (5.6), we have

$$
\begin{aligned}
G\left(g, \frac{1}{a_{i}}\right)= & -\frac{c\left(b_{1}\right)_{1} B_{1}\left(1 / a_{i}\right)}{\left(a_{1}, b_{1} / a_{1}\right)_{1}} \frac{\prod_{s=0}^{m+n}\left(1 / a_{1} q^{s}\right)_{1}}{\prod_{s=0}^{m+n-1}\left(1 / a_{i} q^{s}\right)_{1}} \frac{\bar{P}\left(1 / a_{i}\right) Q\left(q / a_{i}\right)}{P\left(1 / a_{1}\right) \bar{Q}\left(1 / a_{1}\right)} \\
& (i=2, \ldots, N) .
\end{aligned}
$$


Taking the ratio $D_{3}\left(1 / a_{i}\right) / D_{2}\left(1 / a_{1}\right)$ (5.6), we have

$$
\begin{aligned}
G\left(g, \frac{1}{b_{i}}\right)= & -\frac{\prod_{s=0}^{m+n}\left(1 / b_{1} q^{s}\right)_{1}}{\prod_{s=0}^{m+n-1}\left(1 / b_{i} q^{s}\right)_{1}} \frac{\left(a_{1}\right)_{1} A_{1}\left(1 / b_{i}\right)}{\left(a_{1} / b_{1}, b_{1}\right)_{1}} \frac{P\left(q / b_{i}\right) \bar{Q}\left(1 / b_{i}\right)}{\bar{P}\left(1 / b_{1}\right) Q\left(1 / b_{1}\right)} \\
& (i=2, \ldots, N) .
\end{aligned}
$$

Substituting the special values (5.14) into the expressions (5.21)-(5.23) respectively, we obtain the values (5.17)-(5.19).

Remark 5.7 (On the relation between special solutions in Sections 4 and 5). There exists the following relation between the $q$-Appell Lauricella function $\varphi_{D}^{(l)}$ and the $q$-hypergeometric function ${ }_{l+1} \varphi_{l}$ (see $[1,2,9]$ for example):

$$
\begin{aligned}
& { }_{l+1} \varphi_{l}\left(\begin{array}{cc}
a, & b_{1} \ldots, b_{l} \\
& c_{1}, \ldots, c_{l}
\end{array}, u\right) \\
& =\frac{(a u)_{\infty}}{(u)_{\infty}} \prod_{k=1}^{l} \frac{\left(b_{k}\right)_{\infty}}{\left(c_{k}\right)_{\infty}} \varphi_{D}^{(l)}\left(u, \frac{c_{1}}{b_{1}}, \ldots, \frac{c_{l}}{b_{l}}, a u ; b_{1}, \ldots, b_{l}\right) .
\end{aligned}
$$

In view of this, one can expect that the special solutions in Sections 4 and 5 may have some relations. The study of the relations is an interesting future problem.

In [29], some special solution of the higher order $q$-Painlevé system is given in terms of the $q$-hypergeometric function ${ }_{N+1} \varphi_{N}$. Our results suggest the relation between the system in [29] and $q$-Garnier system. In fact, it turns out that these two are equivalent as will be shown in [20].

Acknowledgement. The authors are grateful to Professors Tetsu Masuda, Masatoshi Noumi, Hidetaka Sakai, Takao Suzuki, Takashi Takebe, Teruhisa Tsuda and the referee for stimulating discussions and/or comments. This work was partially supported by JSPS KAKENHI (26287018) and Expenses Revitalizing Education and Research of Akashi College (0216027).

\section{References}

[1] Andrews G. E., Summation and transformation for basic Appell series, J. London Math. Soc., 4 (1972), 618-622.

[2] Andrews G. E., Problems and prospectives for basic hypergeometric functions, in: Theory and application of special functions, Proc. Advanced Sem. Math. Res. Center, University of Wisconsin, Madison, WI, (1975), 19-224.

[ 3 ] Garnier R., Sur des équations différentielles du troisiéme ordre dont l'intégrale générale est uniforme et sur une classe d'équations nouvelles d'ordre supérieur dont l'intégrale générale a ses points critiques fixes, Ann. Sci. Ecole Norm. Super., 29 (1912), 1-126. 
[ 4 ] Gasper G. and Rahman M., Basic Hypergeometric Series. With a foreword by Richard Askey. Second edition. Encyclopedia of Mathematics and its Applications, 96. Cambridge University Press Cambridge, 2004.

[5] Ikawa Y., Hypergeometric solutions for the $q$-Painlevé equation of type $E_{6}^{(1)}$ by the Padé method, Lett. Math. Phys., 103 (2013), 743-763.

[6] Iwasaki K., Kimura H., Shimomura S. and Yoshida M., From Gauss to Painlevé-A modern theory of special functions, Aspects of Mathematics, E16 Vieweg, 1991.

[ 7 ] Jacobi C. G. J., Über die Darstellung einer Reihe gegebner Werthe durch eine gebrochne rationale Function, J. Reine Angew. Math., 30 (1846), 127-156.

[ 8 ] Jimbo M. and Sakai H., A $q$-analog of the sixth Painlevé equation, Lett. Math. Phys., 38 (1996), 145-154.

[9] Kajihara Y., Euler transformation formula for multiple basic hypergeometric series of type $A$ and some applications, Advances in Mathematics, 187 (2004), 53-97.

[10] Kajiwara K., Noumi M. and Yamada Y., Geometric aspects of Painlevé equations, arXiv 1509.08186 [nlin.SI].

[11] Mano T., Determinant formula for solutions of the Garnier system and Padé approximation, J. Phys. A: Math. Theor., 45 (2012), 135206-135219.

[12] Mano T. and Tsuda T., Two approximation problems by Hermite and the Schlesinger transformations (Japanese), RIMS Kokyuroku Bessatsu, B47 (2014), 77-86.

[13] Mano T. and Tsuda T., Hermite-Padé approximation, isomonodromic deformation and hypergeometric integral, Math. Z., 285 (2017), 397-431.

[14] Mumford D., Tata Lectures on Theta, II, Birkhäuser, 1984.

[15] Nagao H., The Padé interpolation method applied to $q$-Painlevé equations, Lett. Math. Phys., 105 (2015), 503-521.

[16] Nagao H., Lax pairs for additive difference Painlevé equations, arXiv:1604.02530 [nlin.SI].

[17] Nagao H., The Padé interpolation method applied to $q$-Painlevé equations II (differential grid version), Lett. Math. Phys., 107 (2017), 107-127.

[18] Nagao H., A variation of the $q$-Painlevé system with affine Weyl group symmetry of type $E_{7}^{(1)}, \quad$ SIGMA, 13 (2017), 092.

[19] Nagao H., Hypergeometric special solutions for $d$-Painlevé equations, arXiv:1706.10101 [nlin.SI].

[20] Nagao H. and Yamada Y., Variations of $q$-Garnier system, arXive:1710.03998 [nlin.SI].

[21] Noumi M., Tsujimoto S. and Yamada Y., Padé interpolation for elliptic Painlevé equation, Symmetries, integrable systems and representations, Springer Proc. Math. Stat., 40 (2013), 463-482.

[22] Quispel G. R. W., Roberts J. A. G. and Thompson C. J., Integrable mappings and soliton equations II, Physica D, 34 (1989), 183-92.

[23] Sakai H., Rational surfaces with affine root systems and geometry of the Painlevé equations, Commun. Math. Phys., 220 (2001), 165-221.

[24] Sakai H., A q-analog of the Garnier system, Funkcialaj Ekvacioj, 48 (2005), 273-297.

[25] Sakai H., Hypergeometric solution of $q$-Schlesinger system of rank two, Lett. Math. Phys., 73 (2005), 237-247.

[26] Sakai H., Lax form of the $q$-Painleve equation associated with the $A_{2}^{(1)}$ surface, J. Phys. A: Math. Gen., 39 (2006), 12203-12210.

[27] Sklyanin E. K., Separation of variables - new trends. Quantum field theory, integrable models and beyond (Kyoto, 1994), Progr. Theoret. Phys. Suppl., 118 (1995), 35-60.

[28] Sklyanin E. K. and Takebe T., Separation of variables in the elliptic Gaudin model, Comm. Math. Phys., 204 (1999), 17-38. 
[29] Suzuki T., A $q$-analogue of the Drinfeld-Sokolov hierarchy of type $A$ and $q$-Painlevé system, AMS Contemp. Math., 651 (2015), 25-38.

[30] Tsuda T., Integrable mappings via rational elliptic surfaces, J. Phys. A: Math. Gen., 37 (2004), 2721-2730.

[31] Tsuda T., On an integrable system of $q$-difference equations satisfied by the universal characters: its Lax formalism and an application to $q$-Painlevé equations, Comm. Math. Phys., 293 (2010), 347-359.

[32] Yamada Y., Padé method to Painlevé equations, Funkcial. Ekvac., 52 (2009), 83-92.

[33] Yamada Y., A Lax formalism for the elliptic difference Painlevé equation, SIGMA, 5 (2009), 042-056.

[34] Yamada Y., Lax formalism for $q$-Painlevé equations with affine Weyl group symmetry of type $E_{n}^{(1)}$, Int. Math. Res. Not 17 (2011), 3823-3838.

[35] Yamada Y., A simple expression for discrete Painlevé equations, RIMS Kokyuroku Bessatsu, B47 (2014), 087-095.

\author{
nuna adreso: \\ Hidehito Nagao \\ Department of Arts and Science \\ National Institute of Technology \\ Akashi College \\ Uozumi, Akashi 674-8501 \\ Japan \\ E-mail: nagao@akashi.ac.jp \\ Yasuhiko Yamada \\ Department of Mathematics \\ Graduate School of Science \\ Kobe University \\ Rokko, Kobe 657-8501 \\ Japan \\ E-mail: yamaday@math.kobe-u.ac.jp
}

(Recevita la 27-an de marco, 2016)

(Revizita la 20-an de decembro, 2017) 